\title{
Consistent comparisons of attainment and shortfall inequality: A critical examination
}

Citation for published version (APA):

Bosmans, K. G. M. (2013). Consistent comparisons of attainment and shortfall inequality: A critical examination. Maastricht University, Graduate School of Business and Economics. GSBE Research Memoranda No. 064 https://doi.org/10.26481/umagsb.2013064

Document status and date:

Published: 01/01/2013

DOI:

10.26481/umagsb.2013064

Document Version:

Publisher's PDF, also known as Version of record

\section{Please check the document version of this publication:}

- A submitted manuscript is the version of the article upon submission and before peer-review. There can be important differences between the submitted version and the official published version of record.

People interested in the research are advised to contact the author for the final version of the publication, or visit the DOI to the publisher's website.

- The final author version and the galley proof are versions of the publication after peer review.

- The final published version features the final layout of the paper including the volume, issue and page numbers.

Link to publication

\footnotetext{
General rights rights.

- You may freely distribute the URL identifying the publication in the public portal. please follow below link for the End User Agreement:

www.umlib.nl/taverne-license

Take down policy

If you believe that this document breaches copyright please contact us at:

repository@maastrichtuniversity.nl

providing details and we will investigate your claim.
}

Copyright and moral rights for the publications made accessible in the public portal are retained by the authors and/or other copyright owners and it is a condition of accessing publications that users recognise and abide by the legal requirements associated with these

- Users may download and print one copy of any publication from the public portal for the purpose of private study or research.

- You may not further distribute the material or use it for any profit-making activity or commercial gain

If the publication is distributed under the terms of Article $25 \mathrm{fa}$ of the Dutch Copyright Act, indicated by the "Taverne" license above, 


\section{Maastricht University}

Kristof Bosmans

Consistent comparisons of attainment and shortfall inequality: A critical examination

RM/13/064

\section{GSBE}

Maastricht University School of Business and Economics

Graduate School of Business and Economics

P.O Box 616

NL- 6200 MD Maastricht

The Netherlands 


\title{
Consistent comparisons of attainment and shortfall inequality:
}

\author{
A critical examination*
}

\author{
Kristof Bosmans \\ Department of Economics, Maastricht University, \\ Tongersestraat 53, 6211 LM Maastricht, The Netherlands
}

October 5, 2013

\begin{abstract}
An inequality measure is 'consistent' if it ranks distributions the same irrespective of whether health quantities are represented in terms of attainment or shortfalls. This consistency property severely restricts the set of admissible inequality measures. We show that, within a more general setting of separate measures for attainments and shortfalls, the consistency property is a combination of two conditions. The first is a compelling rationality condition that says that the attainment measure should rank attainment distributions as the shortfall measure ranks shortfall distributions. The second is an overly demanding condition that says that the attainment measure and the shortfall measure should be identical. By dropping the latter condition, the restrictions on the admissible inequality measures disappear.
\end{abstract}

Keywords. Health inequality · Attainment inequality · Shortfall inequality · Consistency

JEL classification. D39 - D63 · I14

*I am grateful to Guido Erreygers and Erwin Ooghe for extensive comments on an earlier draft. The paper has benefited also from discussions with Oihana Aristondo, Koen Decancq, Casilda Lasso de la Vega and Tom Van Ourti. Remaining shortcomings are mine.

E-mail: k.bosmans@maastrichtuniversity.nl 


\section{Introduction}

Clarke et al. (2002) have shown that conclusions drawn by standard inequality measures may differ depending on whether the individual health data are represented by attainments or by shortfalls to an upper bound. Observations like this have led Erreygers (2009), Lambert and Zheng (2011) and Lasso de la Vega and Aristondo (2012) to search for "consistent' inequality measures, which rank attainment distributions identically as the corresponding shortfall distributions. It has been established that this property - to which we refer as 'strong consistency' - severely restricts the set of admissible inequality measures.

The following example illustrates the limitations imposed by strong consistency. Consider the two-individual attainment distributions $x=$ $(10,70)$ and $y=(30,90)$. With an upper bound of 100 , the corresponding shortfall distributions are $x^{\prime}=(90,30)$ and $y^{\prime}=(70,10)$, respectively. Relative inequality measures, such as the well-known Gini and Theil measures, decrease under equal absolute additions for all individuals. Hence, each relative inequality measure judges $x$ as more unequal than $y$, but $x^{\prime}$ as less unequal than $y^{\prime}$, and thus fails strong consistency. Moreover, the negative implications of strong consistency go much beyond ruling out the relative inequality measures. For example, Lambert and Zheng (2011) show that the variance is the only subgroup decomposable inequality measure that satisfies strong consistency.

We will argue that strong consistency is unduly demanding. The matter is important, as an appropriate weakening of the property turns out to impose no a priori restrictions on the set of admissible inequality measures.

First we focus on the implications of strong consistency. The property aims to capture the rationality idea that inequality judgments should not depend on the arbitrary choice of how to represent the basic data. Therefore, strong consistency should not only apply to attainments and shortfalls, but also to other representations. We show that consistency with respect to one additional representation - in terms of 'relative' shortfalls, which measure the proportional (instead of the absolute) increase required to reach the upper bound - already excludes all relative, absolute and intermediate inequality measures. That is, a direct extension of the logic underlying strong consistency implies the impossibility of inequality measurement using standard methods. This finding encourages scrutinizing the property. 
In order to examine strong consistency, we consider a more general setting that allows separate inequality measures for attainments and shortfalls. Strong consistency may be seen as a combination of two properties, viz., 'weak consistency' and 'uniqueness'. Weak consistency says that the attainment measure should rank attainment distributions in the same way as the shortfall measure ranks the corresponding shortfall distributions. Uniqueness says that the attainment measure and the shortfall measure should be identical. Weak consistency is sufficient to capture the desired rationality requirement, which means that uniqueness is superfluous and strong consistency is too demanding. We show that if weak consistency is imposed without uniqueness, then any relative, absolute or intermediate inequality measure can be chosen for either the attainment or the shortfall measure. This choice then fully determines the properties of the other measure.

The next section introduces notation and basic concepts. Section 3 considers the implications of strong consistency and of its underlying logic. Section 4 examines strong consistency in the general setting with separate attainment and shortfall measures. Section 5 concludes.

\section{Preliminaries}

A distribution is a vector $x=\left(x_{1}, x_{2}, \ldots, x_{n}\right)$ in $\mathbb{R}_{++}^{n}$. The positive real number $x_{i}$ represents the health quantity - e.g., an attainment or a shortfall - of individual $i=1,2, \ldots, n$. For a distribution $x$, we denote the mean $\left(x_{1}+x_{2}+\cdots+x_{n}\right) / n$ by $\bar{x}$. We say that a distribution $x$ is non-equal if not all of the entries of $x$ are equal. We write $1_{n}$ for the $n$-vector with a one at each entry.

An inequality measure is a symmetric and strictly Schur-convex function $I: \mathbb{R}_{++}^{n} \rightarrow \mathbb{R}$ that associates with each distribution $x$ in $\mathbb{R}_{++}^{n}$ an inequality level $I(x)$. Symmetry and strict Schur-convexity ensure that the inequality measure is anonymous (switching individuals' quantities does not change inequality) and satisfies the Pigou-Dalton principle (regressive transfers between individuals increase inequality). These properties are standard in the literature (see, e.g., Cowell, 2000, and Lambert, 2001).

It is common to distinguish inequality measures with respect to their behaviour under equal proportionate and equal absolute increases. We distinguish relative, absolute and intermediate inequality measures. 
(i) An inequality measure $I$ is relative if $I(x)=I(\lambda x)$ for each distribution $x$ and each $\lambda>1$.

(ii) An inequality measure $I$ is absolute if $I(x)=I\left(x+\mu 1_{n}\right)$ for each distribution $x$ and each $\mu>0$.

(iii) An inequality measure $I$ is intermediate if $I\left(x+\mu 1_{n}\right)<I(x)<$ $I(\lambda x)$ for each non-equal distribution $x$, each $\mu>0$ and each $\lambda>1$.

Note that if $I$ is a relative inequality measure, then $I(x)>I\left(x+\mu 1_{n}\right)$ for each non-equal distribution $x$ and each $\mu>0$, and if $I$ is an absolute inequality measure, then $I(x)<I(\lambda x)$ for each non-equal distribution $x$ and each $\lambda>1$ (see, e.g., Moyes, 1999, Proposition 3.4).

Finally, let $b$ be an upper bound on attainments and let $B$ collect each distribution $x$ in $\mathbb{R}_{++}^{n}$ such that $x_{i}<b$ for each individual $i=$ $1,2, \ldots, n$. For a distribution $x$ in $B$, we denote the distribution $(b-$ $\left.x_{1}, b-x_{2}, \ldots, b-x_{n}\right)$ by $b-x$. If $x$ is an attainment distribution, then $b-x$ is the corresponding shortfall distribution. Conversely, if $x$ is a shortfall distribution, then $b-x$ is the corresponding attainment distribution. Note that the set $B$ may be interpreted as the set of all attainment distributions or, alternatively, as the set of all shortfall distributions.

\section{Strong consistency and its implications}

Erreygers (2009) requires an inequality measure to take the same value for an attainment distribution and its corresponding shortfall distribution. Lambert and Zheng (2011) propose a less demanding consistency property. They require that an inequality measure's ranking of two attainment distributions coincides with its ranking of the two corresponding shortfall distributions. We focus on the property of Lambert and Zheng and refer to it as strong consistency. ${ }^{1}$

Strong consistency. For all distributions $x$ and $y$ in $B$, we have

$$
I(x) \leq I(y) \quad \text { if and only if } \quad I(b-x) \leq I(b-y) .
$$

Strong consistency severely restricts the set of admissible inequality measures. Lambert and Zheng (2011, Theorem 3) show that strong consistency is violated by all relative measures and by the particular intermediate measures satisfying Zoli's (1999) 'flexible inequality equivalence'

\footnotetext{
${ }^{1}$ Erreygers' (2009) 'perfect complementarity' property requires $I(x)=I(b-x)$ for each distribution $x$ in $B$. Clearly, perfect complementarity implies strong consistency. Hence, a critique of strong consistency applies also to perfect complementarity.
} 
property. $^{2}$ The following proposition generalizes the result of Lambert and Zheng to all relative and all intermediate inequality measures. ${ }^{3}$ The proof relies on a simple extension of the two-individual example presented in the introduction.

Proposition 1. There is no relative or intermediate inequality measure that satisfies strong consistency.

Proof. Let $b$ be the upper bound on attainments. Let $c, d$ and $e$ be positive real numbers such that $c+d+e<b$. Consider two attainment distributions $x=(c, c, \ldots, c, c+d)$ and $y=(c+e, c+e, \ldots, c+e, c+d+e)$. For each relative and each intermediate inequality measure $I$, we have $I(x)>I(y)$. Now consider the shortfall distributions $b-x=(b-c, b-$ $c, \ldots, b-c, b-c-d)$ and $b-y=(b-c-e, b-c-e, \ldots, b-c-e, b-c-d-e)$. For each relative and each intermediate inequality measure $I$, we have $I(b-x)<I(b-y)$. Hence, each relative and each intermediate inequality measure violates strong consistency.

The fact that strong consistency rules out many interesting inequality measures motivates putting the property under scrutiny. In the next section we will argue that strong consistency is too demanding and that a proper weakening allows far more freedom in choosing inequality measures. But first we look at the implication of extending the logic of strong consistency to a third representation of the basic data.

Consider a representation of a distribution in terms of 'relative' shortfalls. Whereas the shortfall distribution looks at the absolute amount that has to be added to an attainment to reach the upper bound, the relative shortfall distribution looks at the factor by which the attainment has to be multiplied in order to reach the upper bound. For a distribution $x$ in $B$, we denote the distribution $\left(b / x_{1}, b / x_{2}, \ldots, b / x_{n}\right)$ by $b / x$. If

\footnotetext{
${ }^{2}$ An example of a class of intermediate measures that does not fit into Zoli's (1999) mold is that defined by Seidl and Pfingsten (1997).

${ }^{3}$ There do exist non-absolute inequality measures that satisfy strong consistency. Lasso de la Vega and Aristondo (2012) start from a given inequality measure and take the geometric mean of the inequality level of the attainment distribution and the inequality level of the corresponding shortfall distribution. This geometric mean is a new inequality measure that satisfies strong consistency and even Erreygers' (2009) perfect complementarity property. The advantage of this proposal is that any relative, absolute or intermediate inequality measure can be used as a starting point to construct the new inequality measure. The disadvantage is that the new inequality measure itself is not relative, absolute or intermediate, which means it is outside of the standard framework.
} 
$x$ is an attainment distribution, then $b / x$ is the corresponding relative shortfall distribution (and vice versa).

The following property applies the logic of strong consistency to relative shortfalls. It requires the inequality rankings of attainment distributions and the corresponding relative shortfall distributions to be the same.

Strong consistency*. For all distributions $x$ and $y$ in $B$, we have

$$
I(x) \leq I(y) \quad \text { if and only if } \quad I(b / x) \leq I(b / y) .
$$

The next proposition is a counterpart to Proposition 1. The proposition says that strong consistency* excludes all absolute and intermediate inequality measures.

Proposition 2. There is no absolute or intermediate inequality measure that satisfies strong consistency*.

Proof. Let $b$ be the upper bound on attainments. Let $c>0, d>$ 1 and $e>1$ be real numbers such that $c d e<b$. Consider two attainment distributions $x=(c, c, \ldots, c, c d)$ and $y=(c e, c e, \ldots, c e, c d e)$. For each absolute and each intermediate inequality measure $I$, we have $I(x)<I(y)$. Now consider the relative shortfall distributions $b / x=$ $(b / c, b / c, \ldots, b / c, b /(c d))$ and $b / y=(b /(c e), b /(c e), \ldots, b /(c e), c /(c d e))$. For each absolute and each intermediate inequality measure $I$, we have $I(b / x)>I(b / y)$. Hence, each absolute and each intermediate inequality measure violates strong consistency*.

The rationality idea underlying strong consistency requires that the inequality ranking should be the same irrespective of whether the basic data is represented in terms of attainments, (absolute) shortfalls, relative shortfalls, or in any other way. That is, if strong consistency is accepted as a compelling rationality requirement, then so should strong consistency* ${ }^{4}$ But as the following immediate implication of Propositions 1 and 2 shows, this leads to the impossibility of inequality measurement using standard methods. ${ }^{5}$

\footnotetext{
${ }^{4}$ We stress that for our purposes here it is irrelevant whether or not relative shortfalls are used in practice. All that matters is that relative shortfalls constitute another way of representing the same basic data.

${ }^{5}$ Some authors have considered 'super-relative' and 'super-absolute' inequality measures in addition to relative, absolute and intermediate measures (e.g., Amiel and
} 
Corollary. There is no relative, absolute or intermediate inequality measure that satisfies both strong consistency and strong consistency*.

We have looked at the severe implications for inequality measurement of strong consistency and of its underlying logic. Now we turn to a direct examination of the property.

\section{Disentangling strong consistency}

We now consider a more general setting that allows for separate inequality measures for attainments and shortfalls. Thus, we recognize that a priori one may want different properties for an inequality measure depending on what the numbers to which it applies actually mean (e.g., one may want the Pigou-Dalton principle if the numbers are incomes, but not if the numbers are logged incomes). ${ }^{6}$ We denote the attainment inequality measure by $I^{a}$ and the shortfall inequality measure by $I^{s}$. Note that the previous setting with a single measure $I$ for attainments and shortfalls is obtained as a special case by imposing the condition $I^{a}=I^{s}(=I)$. We discuss this condition (the 'uniqueness' property) at the end of this section.

A pair of inequality measures $\left(I^{a}, I^{s}\right)$ consistently compares attainment and shortfall inequality if it satisfies the following property.

Weak consistency. For all distributions $x$ and $y$ in $B$, we have

$$
I^{a}(x) \leq I^{a}(y) \quad \text { if and only if } \quad I^{s}(b-x) \leq I^{s}(b-y) .
$$

Weak consistency is sufficient to guarantee that inequality judgments do not depend on whether the data are represented in terms of attainments or shortfalls. Moreover, it does the job without the two negative implications of strong consistency that were identified in the previous section. To see this, consider the following proposition (of which we omit the simple proof).

Cowell, 1999, Kolm, 1999, and Zheng, 2007). An inequality measure $I$ is super-relative if $I(x)>I(\lambda x)$ for each non-equal distribution $x$ and each $\lambda>1$, and super-absolute if $I(x)<I\left(x+\mu 1_{n}\right)$ for each non-equal distribution $x$ and each $\mu>0$. The proofs of Propositions 1 and 2 can easily be extended to show that each super-relative and each super-absolute inequality measure violates both strong consistency and strong consistency*.

${ }^{6}$ Marchant (2008) stresses this point in the context of currency unit consistency for bankruptcy rules. 
Proposition 3. A pair of inequality measures $\left(I^{a}, I^{s}\right)$ satisfies weak consistency if and only if there exists a strictly increasing function $f$ : $\mathbb{R} \rightarrow \mathbb{R}$ such that

$$
I^{a}(x)=f\left(I^{s}(b-x)\right) \quad \text { for each distribution } x \text { in } B .
$$

First, Proposition 3 reveals that, in contrast to strong consistency (recall Proposition 1 in Section 3), weak consistency does not a priori exclude particular inequality measures. Any relative, absolute or intermediate inequality measure may serve as either the attainment measure or as the shortfall measure. As an example, consider the two inequality measures

$$
B \longrightarrow \mathbb{R}: x \longmapsto \frac{1}{n} \sum_{i=1}^{n} \frac{x_{i}}{\bar{x}} \ln \frac{x_{i}}{\bar{x}}
$$

and

$$
B \longrightarrow \mathbb{R}: x \longmapsto \frac{1}{n} \sum_{i=1}^{n} \frac{b-x_{i}}{b-\bar{x}} \ln \frac{b-x_{i}}{b-\bar{x}} .
$$

The inequality measure in equation (2) is the Theil measure, which is relative and hence excluded by strong consistency. Weak consistency, by contrast, allows choosing this measure as the attainment measure $I^{a}$, provided that (a strictly increasing transformation of) the measure in equation (3) is used as the shortfall measure $I^{s} .^{7}$ Or, conversely, it allows choosing the measure in equation (2) as the shortfall measure $I^{s}$, provided that (a strictly increasing transformation of) the measure in equation (3) is used as the attainment measure $I^{a} .^{8}$ As is clear, the choice of an attainment measure $I^{a}$ fully determines the corresponding shortfall measure $I^{s}$ (up to an increasing transformation), or vice versa. But this should not come as a surprise. Indeed, the rationality idea we aim to capture requires exactly that there is nothing left to be said once the inequality measure for one particular representation of the data is fixed.

\footnotetext{
${ }^{7}$ So, in this case, in equation (2) the set $B$ is the set of all attainment distributions, whereas in equation (3) $B$ is the set of all shortfall distributions.

${ }^{8}$ Indeed, the existence of a strictly increasing function $f$ such that equation (1) holds ensures the existence of a strictly increasing function $g$ (viz., $g=f^{-1}$ ) such that $I^{s}(x)=g\left(I^{a}(b-x)\right)$ for each distribution $x$ in $B$. Incidentally, note that the argumentation of Allanson and Petrie (2012, 2013) and Kjellsson and Gerdtham (2013) may be used to provide a justification for using a measure such as that in equation (3) to measure attainment inequality.
} 
Second, contrary to strong consistency (recall the corollary in Section 3), weak consistency does not become more restrictive if extended to other representations of the data. Consider a general ' $\psi$-representation' that transforms each attainment using a strictly monotonic function $\psi: B \rightarrow$ $\mathbb{R}_{++}$. For an attainment distribution $x$ in $B$, we denote the corresponding ' $\psi$-distribution' $\left(\psi\left(x_{1}\right), \psi\left(x_{2}\right), \ldots, \psi\left(x_{n}\right)\right)$ by $\psi(x)$. For example, we have $\psi(x)=b-x$ in the case of shortfalls and $\psi(x)=b / x$ in the case of relative shortfalls. Consider an attainment measure $I^{a}$ and a measure $I^{\psi}$ defined by

$$
I^{a}(x)=f\left(I^{\psi}(\psi(x))\right) \quad \text { for each distribution } x \text { in } B,
$$

with $f$ a strictly increasing function. We have that $I^{a}$ ranks attainment distributions in the same way as $I^{\psi}$ ranks the corresponding $\psi$ distributions. Clearly, weak consistency can be extended without imposing additional restrictions on inequality measures: the inequality measure for, say, attainments may be chosen freely, and this choice fully determines the inequality measure for each $\psi$-representation.

We conclude this section by clarifying the relationship between weak consistency and strong consistency. The following property on a pair of inequality measures $\left(I^{a}, I^{s}\right)$ says that there should be a unique inequality measure to deal with both attainment and shortfall distributions.

Uniqueness. The inequality measures $I^{a}$ and $I^{s}$ are identical.

As shown by the next proposition (of which we omit the easy proof), strong consistency coincides with the combination of weak consistency and uniqueness.

Proposition 4. A pair of inequality measures $\left(I^{a}, I^{s}\right)$ satisfies weak consistency and uniqueness if and only if there exists an inequality measure $I=I^{a}=I^{s}$ that satisfies strong consistency.

Weak consistency already ensures that inequality judgments do not depend on the chosen representation of the data. Therefore, uniqueness is superfluous and strong consistency is too demanding.

Given that uniqueness is not required for consistent inequality judgments, the question arises whether there are other justifications for the property. A plausible response would be that uniqueness should be seen as a property of convenience. It is indeed convenient not having to change the inequality measure if the representation of the data changes. But this 
mild convenience comes at too high a cost: the propositions and corollary in the previous section amply demonstrate the severely restricting implications of adding uniqueness to weak consistency. ${ }^{9}$

For an alternative possible justification, consider the following scenario. Suppose a practitioner possesses the data for an inequality analysis, but does not possess the information of whether these data are represented in terms of attainments or in terms of shortfalls. Uniqueness is compelling in this case, as it is impossible to determine whether the attainment measure or the shortfall measure should be used. But, of course, the scenario is highly unlikely: precise information on how the data are represented is usually available. However, the scenario does allow another way of seeing why strong consistency is too demanding. Weak consistency allows use of the information on whether numbers express attainments or shortfalls in making consistent inequality judgments. Strong consistency, by additionally imposing uniqueness, does not allow use of this information. But since the information is readily available in practice, this additional restriction is uncalled for.

\section{Conclusion}

We have argued that strong consistency is too demanding. The property combines two conditions. The first is a compelling rationality requirement that states that inequality judgments should not depend on whether the data is represented in terms of attainments or shortfalls. The second is an unnecessarily strong condition that demands that the same inequality measure should be used for attainments and shortfalls. The latter condition may be seen as convenient, but this convenience comes at the high cost of ruling out interesting classes of inequality measures.

An exclusive focus on inequality measures satisfying strong consistency would severely impoverish inequality measurement. It is true that the application of the same, say, relative inequality measure to attainments and to shortfalls yields two different inequality rankings. But these two rankings may each express valid philosophical views on how to make inequality comparisons. ${ }^{10}$ A restriction to inequality measures

\footnotetext{
${ }^{9}$ As Kolm (1976, p. 420) puts it forcefully in the similar context of consistency with respect to different currency units, "convenience could not be an alibi for endorsing injustice".

${ }^{10}$ Allanson and Petrie $(2012,2013)$ and Kjellsson and Gerdtham (2013) also make this point.
} 
that satisfy strong consistency therefore constitutes a partial neglect of the spectrum of valid views.

A final word of caution is in order. The temptation should be resisted to blindly apply the well-known inequality measures to any of the numerous possible representations of the basic data. The properties one wants for an inequality measure are likely to depend crucially on the specific way in which the data are represented. Hence, a study of inequality should ideally motivate both the choice of the particular representations of the data focused on and the choice of the inequality measures applied to each of these representations.

\section{References}

Allanson P and D Petrie, 2012. Understanding the vertical equity judgements underpinning health inequality measures. Discussion Papers 264, University of Dundee.

Allanson P and D Petrie, 2013. On the choice of health inequality measure for the longitudinal analysis of income-related health inequalities. Health Economics 22, 353-365.

Amiel Y and FA Cowell, 1999. Income transformations and income inequality. In: D Slottje (ed), Advances in Econometrics, Income Distribution and Scientific Methodology. Heidelberg: Physica Verlag, 209-232.

Clarke PM, U-G Gerdtham, M Johannesson, K Bingefors and L Smith, 2002. On the measurement of relative and absolute income-related health inequality. Social Science \& Medicine 55, 1923-1928.

Cowell FA, 2000. Measurement of inequality In: A Atkinson and F Bourguignon (eds), Handbook of Income Distribution I. Amsterdam: Elsevier, 87-166.

Erreygers G, 2009. Can a single indicator measure both attainment and shortfall inequality? Journal of Health Economics 28, 885-893.

Kjellsson G and U-G Gerdtham, 2013. Lost in translation. Rethinking the inequalityequivalence criteria for bounded health variables. Working Paper 2013:18, Lund University.

Kolm S-C, 1976. Unequal inequalities I. Journal of Economic Theory 12, 416-442.

Kolm S-C, 1999. The rational foundations of income inequality measurement. In: J Silber (ed), Handbook of Income Inequality Measurement. Dordrecht: Kluwer, 19-100.

Lambert P, 2001. The Distribution and Redistribution of Income: A Mathematical Analysis. Third edition. Manchester: Manchester University Press

Lambert P and B Zheng, 2011. On the consistent measurement of attainment and shortfall inequality. Journal of Health Economics 30, 214-219.

Lasso de la Vega C and O Aristondo, 2012. Proposing indicators to measure achievement and shortfall inequality consistently. Journal of Health Economics 31, 578-583. 
Marchant T, 2008. Scale invariance and similar invariance conditions for bankruptcy problems. Social Choice and Welfare 31, 693-707.

Moyes P, 1999. Stochastic dominance and the Lorenz curve. In: J Silber (ed), Handbook of Income Inequality Measurement. Dordrecht: Kluwer, 199-222.

Seidl C and A Pfingsten, 1997. Ray invariant inequality measures. In: S Zandvakili (ed), Research on Economic Inequality, vol. 7. Greenwich: JAI Press, 107-129.

Zheng B, 2007. Unit-consistent decomposable inequality measures. Economica 74, 97111.

Zoli C, 1999. A generalized version of the inequality equivalence criterion: a surplus sharing characterization, complete and partial orderings. In: H de Swart (ed), Logic, Game Theory and Social Choice: Proceedings of the International Conference LGS'99. Tilburg: Tilburg University Press, 427-441. 